\title{
Experimental Investigation of Turbulence Diffusion-A Factor in Transportation of Sediment in Open-Channel Flow
}

\author{
By E. R. VAN DRIEST,2 PASADENA, CALIFORNIA
}

Turbulence diffusion in open-channel flow was investigated experimentally by photographing the spread of globules formed by the injection of an immiscible fluid into water. The mean-square transverse deviations of the globules at various distances downstream from the source were computed and analyzed in an effort to determine the shape of the velocity-correlation curve. Comparison was made between two types of curve which fitted the deviation data, one corresponding to a power-correlation law and the other to an exponential-correlation law.

\section{INTRODUCTION}

$\mathrm{T}$ URBULENCE diffusion in flowing water has for a long time been of interest to the fluid technician and the hydraulic engineer. Inherent in the turbulence of the water, this diffusion is responsible for the existence of many internal phenomena such as eddy heat transfer and sediment suspension. Although the findings of the investigation will be of interest in many fields, this work was undertaken primarily because of its importance in sediment suspension and sediment transportation.

Diffusion in water may be characterized by the diffusion coefficient. A detailed experimental investigation of this coefficient is necessary therefore in order to understand more completely the diffusion mechanism of open-channel flow and to apply it to the various diffusion phenomena. Of particular importance is a knowledge of the functional variation of the diffusion coefficient from point to point, for without that, integration of the fundamental equation for sediment suspension is impossible.

Some observations $(1)^{3}$ on diffusion in water have been made, but most investigations have been conducted in air, either in the wind tunnel (2) or in the atmosphere (3).

\section{THEORY}

The diffusion theory for the turbulent motion of a fluid is closely analogous to the theory of the diffusion of gases and of particles in the Brownian movement. For this reason the latter theory will be reviewed first.

${ }_{1}$ Published with the permission of the United States Department of Agriculture, Soil Conservation Service, H. H. Bennett, Chief.

2 Ph.D. Thesis, 1940, California Institute of Technology; now Assistant Professor of Mechanical Engineering, Massachusetts Institute of Technology, Cambridge, Mass, Mem. A.S.M.E.

Numbers in parentheses refer to the Bibliography at the end of the paper.

Contributed by the Applied Mechanics Division and presented at the Annual Meeting, New York, N. Y., Nov. 27-Dec. 1, 1944, of The American Society of Mechanical Engineers.

Discussion of this paper should be addressed to the Secretary, A.S.M.E., 29 West 39th Street, New York, N. Y., and will be accepted until July 10, 1945, for publication at a later date. Discussion received after the closing date will be returned.

Note: Statements and opinions advanced in papers are to be understood as individual expressions of their authors and not those of the Society.

\section{Molecular Diffusion-Kinetic Theory}

In the kinetic theory of gases the fundamental equation for onedimensional steady-state molecular diffusion is given by

$$
n=-D \frac{d N}{d x} \ldots \ldots \ldots \ldots \ldots \ldots \ldots \ldots
$$

where $n$ is the time rate of diffusion of certain selected molecules through a unit area normal to the $x$ axis and in the positive direction of this axis, $N$ is the concentration of such molecules at the point in question, and $D$ is the diffusion coefficient. Then, if $N$ is a function of time and space, and $D$ is allowed to vary from point to point, it can readily be shown that the general equation for diffusion of certain molecules in a laminar gas stream with compressibility neglected is

$$
\begin{array}{r}
\frac{\partial N}{\partial t}+U \frac{\partial N}{\partial x}+V \frac{\partial N}{\partial y}+W \frac{\partial N}{\partial z}=\frac{\partial}{\partial x}\left(D \frac{\partial N}{\partial x}\right)+\frac{\partial}{\partial y}\left(D \frac{\partial N}{\partial y}\right) \\
+\frac{\partial}{\partial z}\left(D \frac{\partial N}{\partial z}\right) .[2]
\end{array}
$$

In this expression $U, V$, and $W$ are the components of fluid velocity in the $x-, y$-, and $z$-directions, respectively. If the fluid is at rest, and $N$ and $D$ are functions of $y$ only, Equation [2] reduces to the one-dimensional case

$$
\frac{\partial N}{\partial t}=D \frac{\partial^{2} N}{\partial y^{2}}
$$

It is next assumed that Equation [1] holds for very small solid particles suspended in a liquid by molecular collision (Brownian movement) and subjected to an extraneous field of force such as gravity. If the particles have a mean settling velocity $\eta$, and the $y$-direction is upward, Equation [3] must be rewritten to read

$$
\frac{\partial N}{\partial t}=\eta \frac{\partial N}{\partial y}+D \frac{\partial^{2} N}{\partial y^{2}}
$$

and in the steady state

$$
\eta N=-D \frac{d N}{d y}
$$

Equation [4] is the law governing the suspension of minute particles in a liquid at rest, suspension being due solely to molecular collision. The law was verified experimentally by Perrin (4) in 1908.

In the self-diffusion of gases, a derivation by Jeans (5) gives

$$
D=\frac{1}{3} \bar{c} L \ldots \ldots \ldots \ldots \ldots \ldots \ldots
$$

where $\bar{c}$ is the mean velocity of the gas molecules and $L$ is their mean free path. Here it is supposed that the molecules passing through a plane have traveled, on the average, uninterruptedly from various regions at distances perpendicular to the plane 


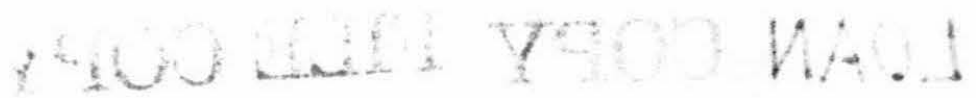

equal to the projections of the mean free path of the molecules. On the other hand, in the Brownian movement, the coefficient of diffusion may be determined by measurement of displacement of the particles in an interval of time. Einstein (6) proved that for such particles (or molecules)

$$
D=\frac{1}{2} \frac{\overline{Y^{2}}}{\tau} .
$$

in which $\overline{Y^{2}}$ is the mean-square displacement of the particles in the $y$-direction during an interval of time $\tau$. It is assumed that $\tau$ is of such magnitude that successive movements of a particle are randomly distributed and independent of each other. In other words, the interval $\tau$ is sufficiently long to insure that the effects on displacement of correlation of velocities after successive bumps are negligible.

An important feature of the diffusion of molecules is the transportation of energy and momentum as well as of mass of the molecules. However, the idea alone of movement of molecules from one region to another is not sufficient to explain the distribution of mean temperature or velocity in the parallel flow of a nonturbulent gas; it is necessary to assume, furthermore, that, upon arrival of a molecule at a new layer, the molecule gives up the excess or takes on the deficiency of mean energy or momentum which it had at the old layer where it started its path. The distance between the layers for each excursion of a typical molecule is taken as the projection of the mean free path on the line normal to the layers. The effect of energy transport in this manner is heat conduction; thus if temperature is substituted for concentration in Equation [2], there results the heat-conduction equation for a moving gas when compressibility and dissipation are neglected (this equation also holds for heat conduction in a liquid stream). The effect of transfer of momentum is the wellknown phenomenon called viscosity.

\section{Turbulence Diffusion-Transfer Phenomena}

Fluids in turbulent motion exhibit a turbulence diffusion in addition to a diffusion due to molecular activity. By analogy with the kinetic theory of gases, the concept of a "mixing" process coincident with the diffusion of particles of fluid has been brought forward to account for the distribution of mean properties of a fluid in a turbulent field. In the so-called mixing process, properties of a fluid are supposedly transferred by the motion of small fluid masses each of which moves from one layer in which its property is that of the mean flow in the neighborhood to another layer at a transverse distance $\lambda$. After each mass has moved a distance $\lambda$, retaining its original property, it is supposed to mix with the surrounding fluid until its initial identity is lost, thereby assuming the average property of the new region. Transferable properties of a fluid may be internal energy, salinity, concentration of sediment, etc. Momentum has been assumed such a property, giving rise to eddy viscosity, analogous to molecular viscosity in the kinetic theory of gases.

Now it is readily shown (7) that the fundamental equation for the mean rate of turbulence transfer $m$ of any local property $M$ through a unit area normal to the $y$ axis in the positive direction is given by

$$
m=-\overline{v \lambda} \frac{d M}{d y} \ldots \ldots \ldots \ldots \ldots \ldots \ldots[7]
$$

in which $v$ is the instantaneous transverse-velocity fluctuation, the bar indicating the mean value over the plane surface in question. In correspondence with the molecular theory, $\bar{v}$ is called the diffusion or transfer coefficient. If this mean product is written as the product of a mean velocity and a mean length, thus

$$
\overline{v \lambda}=\sqrt{\overline{v^{2}} l} \ldots \ldots \ldots \ldots \ldots \ldots[8]
$$

$l$ becomes, by definition of Equation [8], the effective mean length of travel of a fluid mass before it loses its property identity in the surrounding fluid. In this manner $l$ may be defined as the mixing length. It is analogous to the mean free path of molecular diffusion. If sediment of concentration $N$ and fall velocity $\eta$ is under consideration, then, upon equilibrium, there results an equation identical with Equation [4] for the suspension of sediment due to turbulence.

There is some question as to whether $\sqrt{\overline{v^{2}}} l$, or rather $l$, is the same for the transfer of different properties. Thus far, some experiments ( 8 ) have been conducted to compare the transfer coefficients for momentum and sediment transfer.

In contrast with the discontinuous mixing process in which a lump of fluid moves a distance and then mixes with its surroundings, Taylor (9) has developed a theory of diffusion in which no assumption of mixture is made. Taylor showed that it is again possible to derive an equation similar to Einstein's Brownianmovement formula, Equation [6], thus

$$
D=\frac{1}{2} \frac{\overline{Y^{2}}}{\tau} \text {. }
$$

where $\overline{Y^{2}}$ is the mean-square displacement of fluid particles and $\tau$ is again an interval of time of such duration that the influence of correlation of successive veloeities upon the observations is negligible.

\section{Turbulence Diffusion-Continuous Movements}

In Taylor's development of a theory for turbulence diffusion, cognizance is taken of the fact that the diffusion process is not a discontinuous one, but rather a continuous one, since the velocities and movements of the particles are continuous. This idea of continuity of motion is the essential difference between Taylor's theory and the transfer theory, as well as the molecular theory.

In order to describe the continuous motion of particles of fluid, Taylor has introduced the statistical correlation between successive velocities of a particle of fluid. The development is as follows:

Consider the random migration of particles of fluid in the $y$ direction and let $v$ be the instantaneous fluctuating velocity parallel to that direction. The correlation coefficient $R \xi$ between the velocity of a particle of fluid at time $t$ and the velocity of the same particle after an interval of time $\xi$ is given by

$$
R \xi=\frac{\overline{v_{t} v_{t+\xi}}}{\sqrt{\overline{v_{t}^{2}}} \sqrt{\overline{v_{t+\xi^{2}}}}} \cdots \ldots \ldots \ldots \ldots \ldots[10]
$$

If the turbulence is considered uniform with respect to space and time, and $R_{\xi}$ is an even function of $\xi$, then

and

$$
\sqrt{\overline{v_{t}^{2}}}=\sqrt{\overline{\overline{v_{t+\xi^{2}}}}}=\sqrt{\overline{\overline{v_{t-\xi^{2}}}}}
$$

$$
R \xi=\frac{\overline{v_{t} v_{t-\xi}}}{\overline{v_{t}^{2}}}
$$

The value of the definite integral $\int_{0}^{t} \overline{v_{t} v_{t-\xi}} d \xi$ is next considered, whence by the definition of the correlation coefficient there follows

Now

$$
\int_{0}^{t} \overline{v_{t} v_{t-\xi}} d \xi=\int_{0}^{t} R_{\xi v_{t}^{2}} d \xi=\overline{v_{t}^{2}} \int_{0}^{t} R_{\xi} d \xi \ldots \ldots \ldots
$$

$$
\int_{0}^{t} \overline{v_{t} v_{t-\xi}} d \xi=\overline{v_{t} \int_{0}^{t} v_{t-\xi} d \xi}=\overline{v_{t} Y}=\frac{1}{2} \frac{d \overline{Y^{2}}}{d t}
$$


where $Y$ is the displacement of a particle in time $t$, so that by Equation [11]

$$
\overline{v^{2}} \int_{0}^{t} R_{\xi} d \xi=\overline{v_{t} Y}=\frac{1}{2} \frac{d \overline{Y^{2}}}{d t}: \ldots \ldots \ldots \ldots \ldots
$$

integration of which yields

$$
\overline{Y^{2}}=2 \overline{v^{2}} \int_{0}^{T} \int_{0}^{t} R_{\xi} d \xi d t .
$$

It is therefore seen that diffusion in a field of uniform turbulence is dependent upon two quantities, i.e., the energy of turbulence, and the coefficient of correlation $R \xi$.

Equation [13] offers a means of measuring the intensity of turbulence $\sqrt{\overline{v^{2}}}$. By observing the displacement of particles so close to a point source that $R_{\xi} \rightarrow 1$, it follows that

$$
\overline{Y^{2}}=\overline{v^{2}} T^{2}
$$

or

$$
\sqrt{\overline{v^{2}}}=\frac{\sqrt{\overline{Y^{2}}}}{T}
$$

which leads to

$$
\sqrt{\overline{v^{2}}}=\frac{\sqrt{\overline{Y^{2}}}}{x} U
$$

if the point is fixed and the fluid is moving with the mean velocity $U$ in a direction $x$ transverse to the migration.

Of more importance is the case when $R_{\xi} \rightarrow 0$. If it can be assumed that such a time interval $T_{1}$ exists that the velocity of a particle at the end of the interval $T_{1}$ has no correlation with the velocity at the beginning of the interval, then $\int_{0}^{T_{1}} R \xi d \xi$ is finite, and

$$
\overline{v^{2}} \int_{0}^{T_{1}} R_{\xi} d \xi=\overline{v_{t} Y}=\text { const. }
$$

for all values of $T>T_{1}$. Hence $T_{1}$ is the true average time required for a particle to lose its velocity identity in the surrounding fluid.

According to the condition of Equation [15], it is possible to define a length $l_{1}$ by the relation

$$
l_{1}=\sqrt{\overline{v^{2}}} \int_{0}^{T_{1}} R_{\xi} d \xi
$$

so that

$$
\sqrt{\overline{v^{2}}} l_{1}=\overline{v_{t} Y}=\frac{1}{2} \frac{d \overline{Y^{2}}}{d t}=\frac{U}{2} \frac{d \overline{Y^{2}}}{d x} .
$$

whence

$$
\overline{Y^{2}}=2 \sqrt{\overline{v^{2}}} l_{1} T+\text { const. }
$$

or

$$
\overline{Y^{2}}=\frac{2 \sqrt{\overline{v^{2}} l_{1}}}{U} x+\text { const. }
$$

which is the equation of the straight line to which the $\overline{Y^{2}}$ diffusion curve becomes tangent.

It can be seen by Equation [18] that, when $T$ is sufficiently large, $\overline{Y^{2}}$ becomes directly proportional to time $\tau$ as mentioned previously in Equation [9], thus

$$
\overline{Y^{2}}=2 \sqrt{\overline{v^{2}}} l_{1} \tau
$$

Comparison of Equation [20] for turbulence diffusion with Einstein's Equation [6] for the Brownian movement readily indicates that $\sqrt{\overline{v^{2}}} l_{1}$ may be called the turbulence-diffusion coefficient with $l_{1}$ corresponding to the mean free path $L$ in the case of molecular diffusion.
It follows by Equation [16] that the diffusion length $l_{1}$ is, like the mixing length, some mean effective transverse distance which a particle of fluid travels before losing its identity in the fluid neighborhood, but it is to be noted that here the specific identity is that of velocity and not necessarily that of any property. It might also be agreed by Equation [16] that $\int_{0}^{T_{1}} R \xi d \xi$ is the effective average time required for a particle to lose its velocity identity as compared to $T_{1}$, which is the true average time required for such a loss.

Equation [17] shows that the diffusion coefficient can be determined by direct measurement of the slope of the mean-square deviation line when data are taken at times longer than that for which $R_{\xi} \rightarrow 0$. However, sometimes it may be difficult to obtain diffusion data for such a direct measurement, since $T_{1}$ may be quite large. This is in contrast with measurements in the Brownian movement, where it is hardly possible to make observations at intervals of time so short that persistence of velocities would affect successive displacements.

\section{Correlation}

Since the correlation between successive velocities of a fluid particle has been shown to play an important part in the development of the theory of turbulence diffusion, further discussion of the meaning of correlation is desirable.

The term correlation refers generally to the statistical interdependence of variables, whereas the coefficient of correlation is a statistic which is a numerical measure of the degree of interdependence of variables. The coefficient as used previously (Equation [10]) is defined as the algebraic-mean value of the product of successive velocities of a particle divided by the product of the root-mean-square values of the fluctuations at the beginning and end of the time interval; of course, a sufficient number of observations are necessary to insure a true mean. Fig. 1 shows schematically various degrees of correlation between velocities, the high correlation corresponding to a short time interval, the low corresponding to a long time interval. Other types of correlation $(10,11)$ exist between velocities in a turbulent field.

There is one important limitation to the correlation coefficient as previously defined; it can be applied only to data which scatter about a straight line. However, in correlating velocities of the same particle the regression is assumed to be linear, and
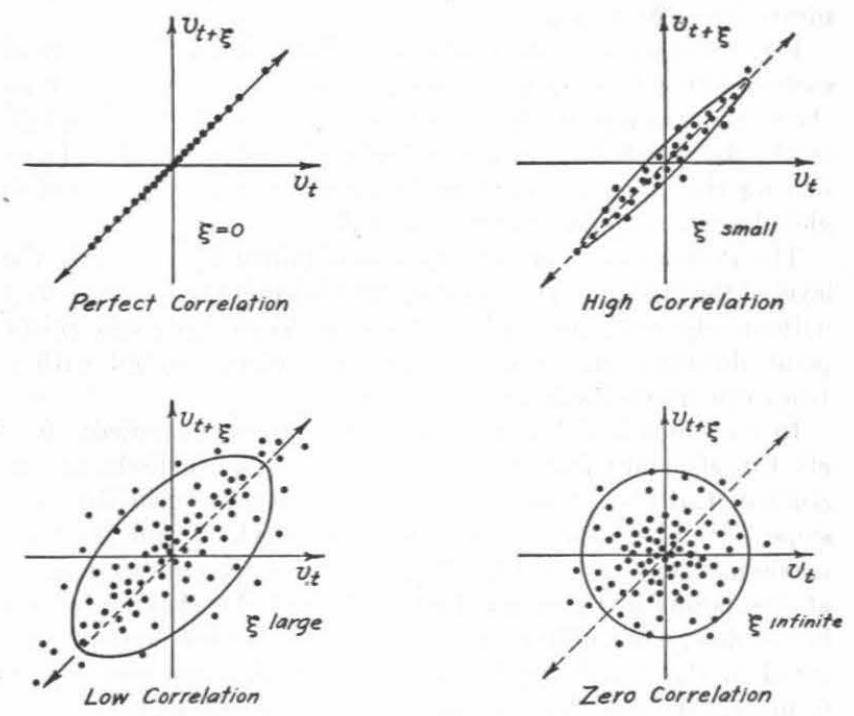

Fig. 1 Schematic Representation of Correlation of Successive Velocities of a Fluid Particle 


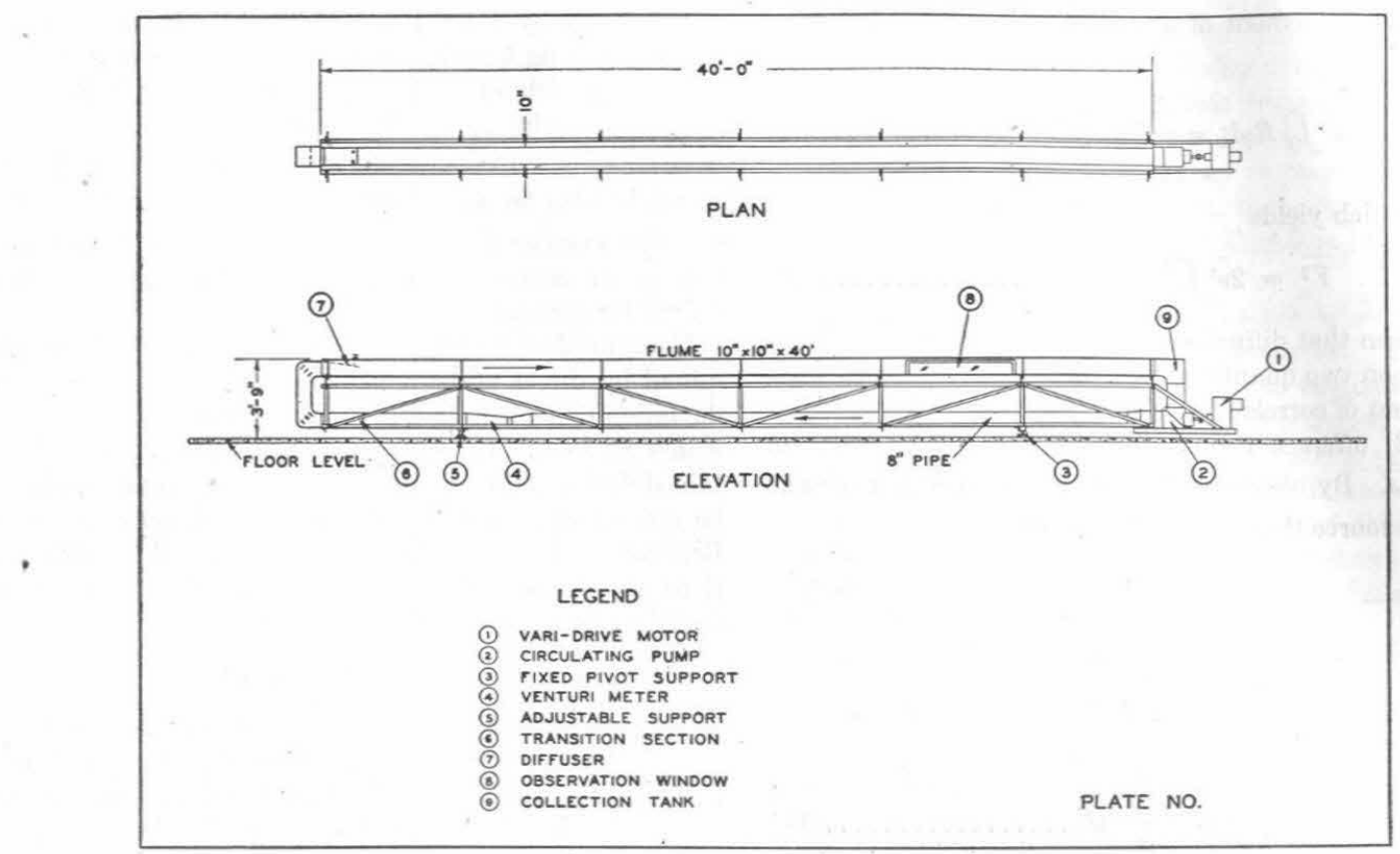

Fig. 2 Diagrammatic Sketch of 10-In. Closed-Circuit Flume

there is no reason to believe that in the random fluctuations, which are encountered in a uniformly turbulent field, the trend will be anything but linear.

\section{LABORATORY EXPERIMENTS}

The experiments were conducted in a closed-circuit flume (see Figs. 2 and 3), $10^{1 / 2}$ in. wide, $10 \mathrm{in}$. deep, and $40 \mathrm{ft}$ long. The slope could be adjusted by means of a jack-and-pivot combination. On either side of the flume was a glass window $5 \mathrm{ft}$ long and $7 \mathrm{ft}$ from the downstream end. Also at the downstream end was a motor-and-propeller-pump assembly, the discharge of which was measured by a Venturi meter. Two channel conditions were used in the investigation, smooth walls and bottom, and rough walls and bottom. Smooth surfaces were obtained by the application of bitumastic paint; rough surfaces were produced by covering soft bitumastic paint with sand grains having a mean size of $0.89 \mathrm{~mm}$.

Fig. 4 shows the injection apparatus in position. A mixture of carbon tetrachloride and benzene, having the same density as the water, was injected into the flow through a 24 -gage stainlesssteel tube which had an internal diameter of 0.0123 in. Upon leaving the tube, the mixture broke up into small, immiscible globules which could be photographed.

The proper injection velocity was obtained by adjusting the level of the fluid in a burette tube. The injection tube was aligned with an adjustable index plate which was located at a convenient point downstream. The globules were photographed with a Leica camera on Kodak Plus-X film.

In the smooth channel, three discharges were studied: 0.58 cfs, $1.08 \mathrm{cfs}$, and $1.76 \mathrm{cfs}$; in the rough channel, one discharge was atudied, $1.51 \mathrm{cfs}$. The flow in the rough channel had the same slope as the high flow in the smooth channel. In all flows the maximum depth was held at $0.65 \mathrm{ft}$. Measurements were made at five points in the vertical center line of the channel for the lowest flow, while only two points in the center line were investigated in the remaining flows. The Reynolds numbers ranged from 27,200 to 82,500 using the hydraulic radius as the characteristic length.

A particular discharge and depth of water at uniform flow were

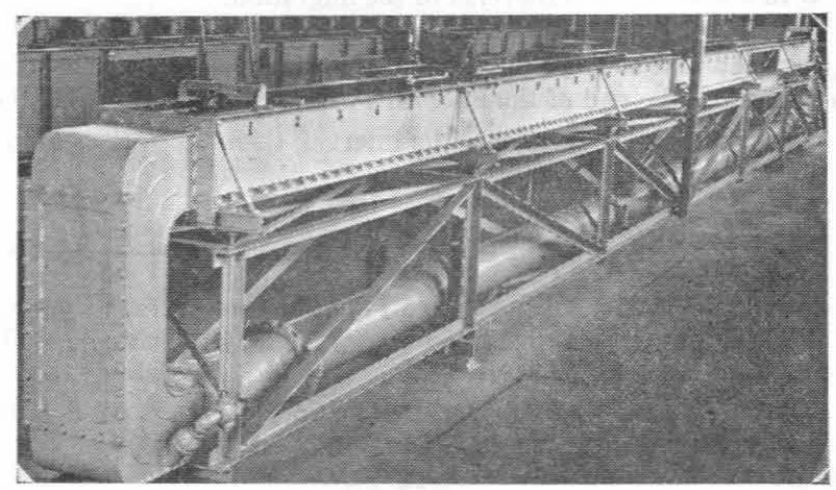

Fig. 3 Side View of Flume Showing Adjustable and Pivot Supports, Observation Window, and Venturi Meter

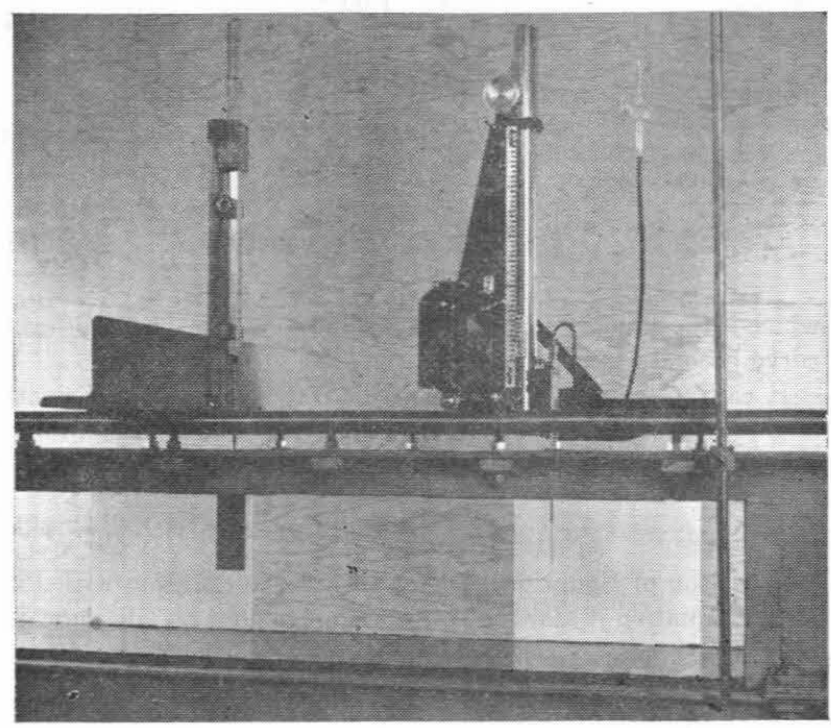



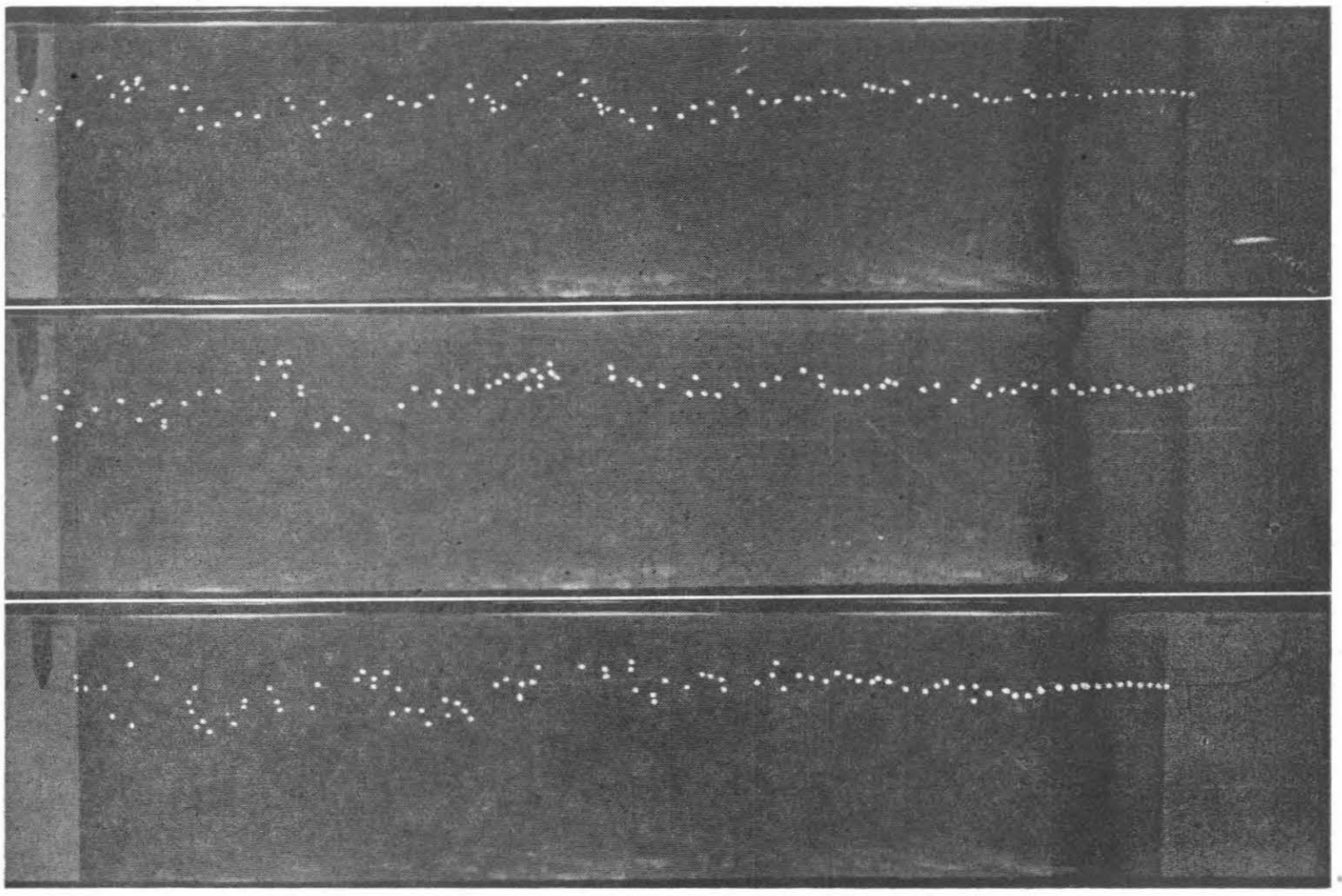

Fig. 5 Typical Diffusion Photographs (Note the periodic wandering which gives some indication of a definite turbulence pattern. The globules have been touched up for

established by adjusting the slope of the flume and speed of the pump. After the flow became steady, the immiscible fluid was injected and about thirty photographs (one roll) were taken in rapid succession. Four or five rolls of film were used for each depth in a particular flow. After each roll of photographs had been exposed, the water was freed of globules by circulating it through two layers of cheese cloth. Fig. 5 shows reproductions of three typical instantaneous photographs of diffusion at the same injection depth and discharge.

After development, the films were projected on a screen to exactly full size. At various distances downstream from the injector tube, the vertical positive and negative displacements within a narrow band were read off the screen to the nearest $0.01 \mathrm{in}$. by means of co-ordinate paper. For the study of the two lower discharges, displacements were obtained at the following downstream points: $1 / 4,1 / 2,1,1 \frac{1}{2}, 2,3,-13$ in., and as far as $30 \mathrm{in}$. at one depth. For the higher discharges, the following points were used: $1 / 8,1 / 4,1 / 9,1,1^{1} / 2,2,3,-13$ in., or $1 / 8,1 / 4$, $1 / \mathrm{s}, 1,1^{1} / 2,2,3,4,6,8,-14$ and, in one case to $32 \mathrm{in}$. downstream. The mean-square values of these displacements were then calculated at the various distances from the point source, each value arrived at representing the mean of about 400 measurements.

\section{ANALYSIS OF DATA}

\section{Power-Correlation Law}

The mean-square deviation data show considerable scatter when plotted on Cartesian co-ordinate paper, Fig. 6, despite the great number of measurements taken for each mean. Beoause of this scatter, the author was not able to measure directly the slope of the limiting deviation line to determine the diffusion coefficient. If, however, the data are plotted on log-log paper, Figs. 7 to 10 , they are found to adhere closely to a straight line, except near the origin, the straight-line plot meaning that the data in that range may be approximated by a power law

$$
\overline{Y^{2}}=C x^{n} \text {. }
$$

where $n$ must lie between 2 and 1, as required by Equations [14] and [19], respectively. The fitted curves, obtained from the logarithmic plot in Fig. 7, are transferred to the Cartesian paper, as shown in Fig. 6.

The adoption of such a power law to represent the data allows the calculation of the correlation coefficient from

$$
R_{\xi}=\frac{1}{2}\left(\frac{U}{\sqrt{\overline{v^{2}}}}\right)^{2} \frac{d^{2} \overline{Y^{2}}}{d x^{2}} .
$$

which is obtained upon differentiation of Equation [12] and use of $x=U t$. Thus upon substitution of Equation [21] into Equation [22], there is obtained

$$
R_{\xi}=\frac{1}{2}\left(\frac{U}{\sqrt{v^{2}}}\right)^{2} C n(n-1) x^{n-2} .
$$

It is seen that the use of this equation requires an independent determination of $\frac{\sqrt{\overline{v^{2}}}}{U}$, which is obtained from the slope of the $\overline{Y^{2}}$ curve at the origin through Equation [14].

While Equation [23] indicates a possible shape of the correlation curve, its chief drawback is that its integral is infinito, 

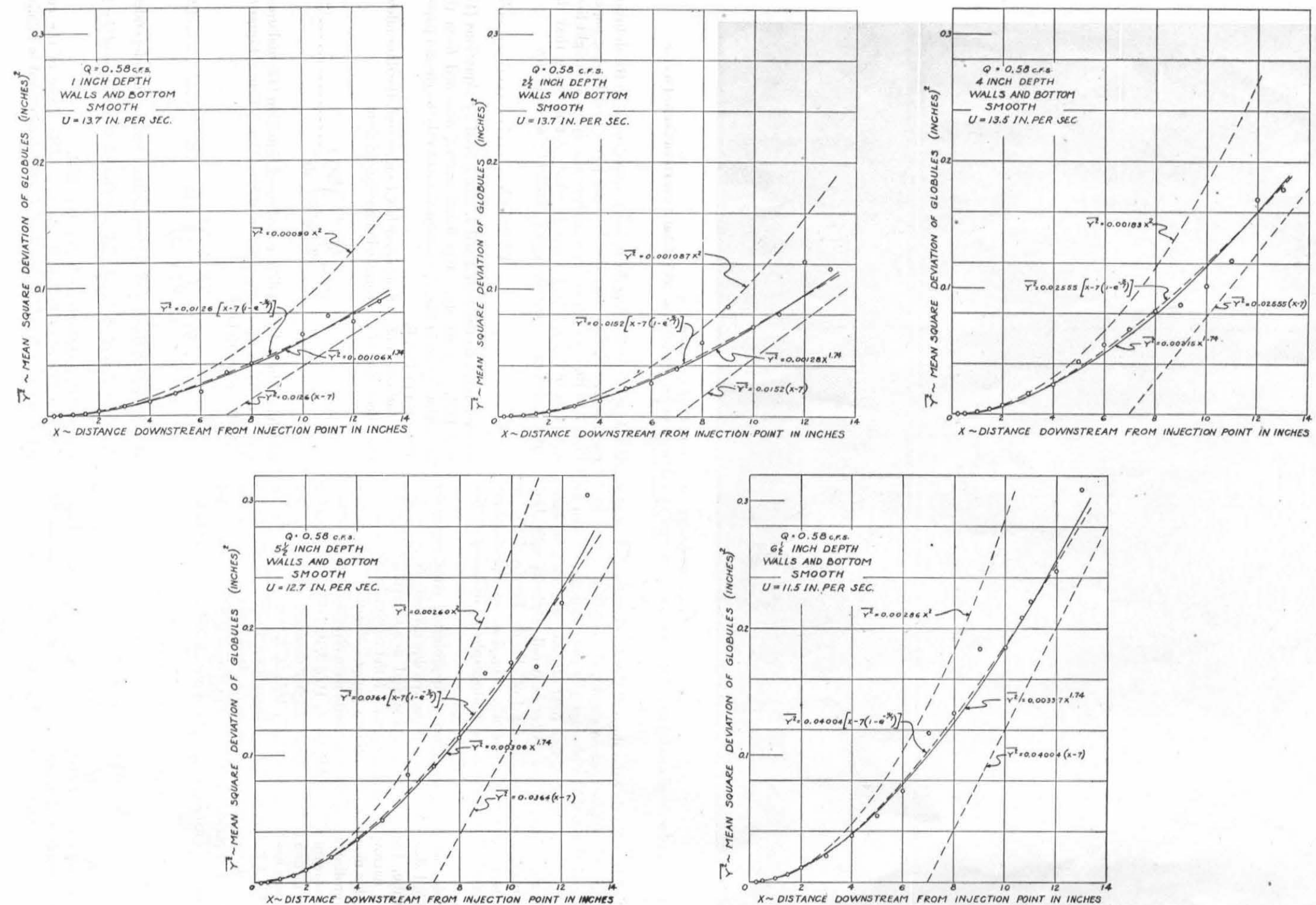

Fig. 6 Mean-Square Vertical Deviation Curves at $1,2 \frac{1}{2}, 4,5^{1 / 2}$, and $6 \frac{1}{2}-$-In. Depths for $Q=0.58$ Cfs (Walls and bottom smooth.) 


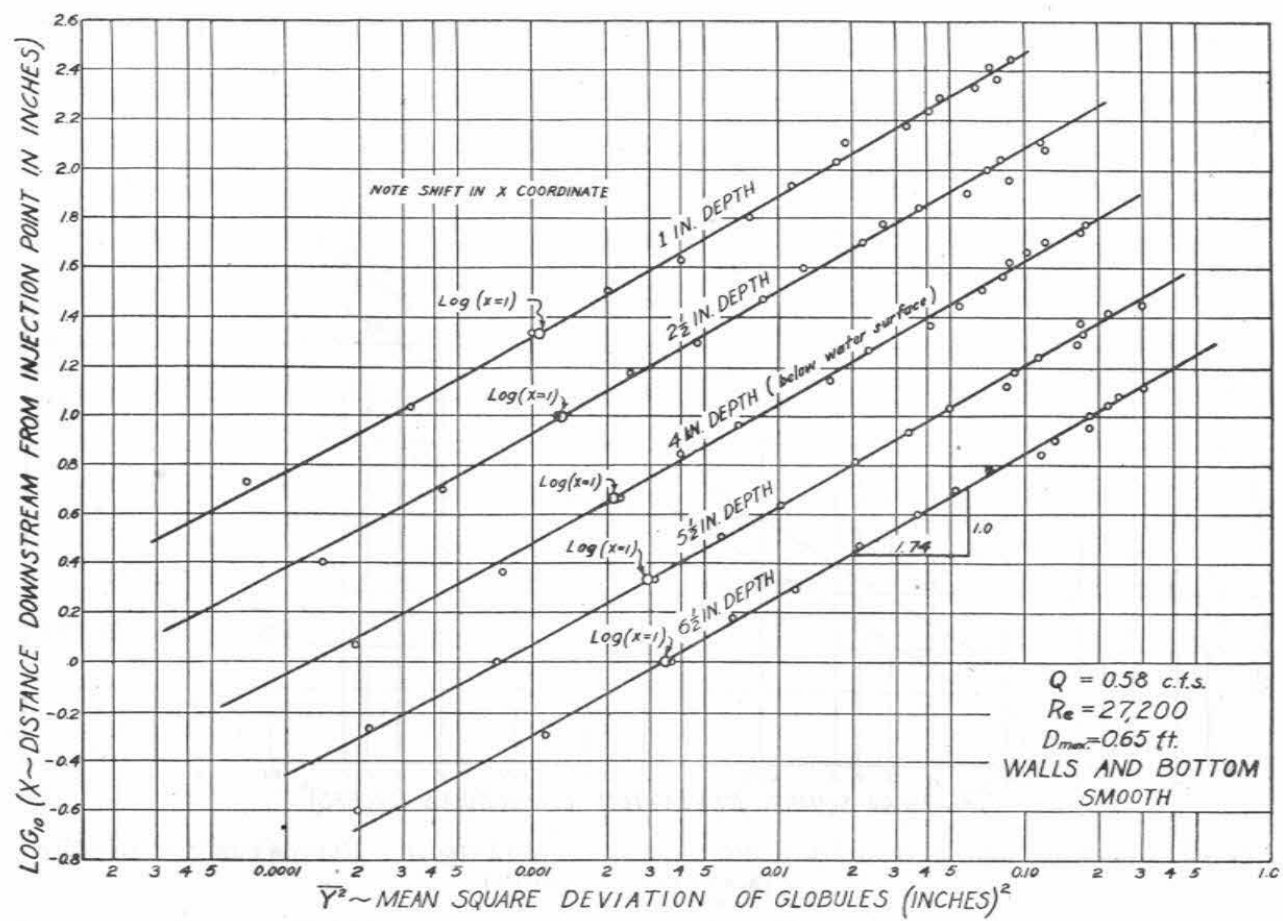

Fig. 7 Mean-Square Vertical Deviation Curves at Various Injection Depths for $Q=0.58$ Cfs (Walls and bottom smooth.)

which leads to an infinite diffusion length $l_{1}$ and, consequently, an infinite diffusion coefficient. In the case of the open-channel flow investigated herein, such a condition is not expected to exist, since the size of the eddies is limited by the width of the channel, and it is expected that the correlation of successive velocities would approach zero rapidly after a particle of fluid was carried about by a number of eddies.

However, a condition in which the diffusion length $l_{1}$ may become indefinitely large is that which exists in the atmosphere where there is no a priori reason that the eddies should have a limiting size. This case was investigated by Sutton (12), using data collected by Richardson and Proctor (3) on the diffusion of balloons in the atmosphere. Sutton plotted the data on loglog paper and found that a linear variation with $n=1.75 \mathrm{ap}-$ proximated the data, a perfect agreement with the author's 1.74 in the low flow, Fig. 7. It is to be noted, however, that the values for the author's remaining flows do not agree with Sutton's value.

Of interest is the fact that in each flow the straight-line portions of the curves are parallel. This means, of course, that the

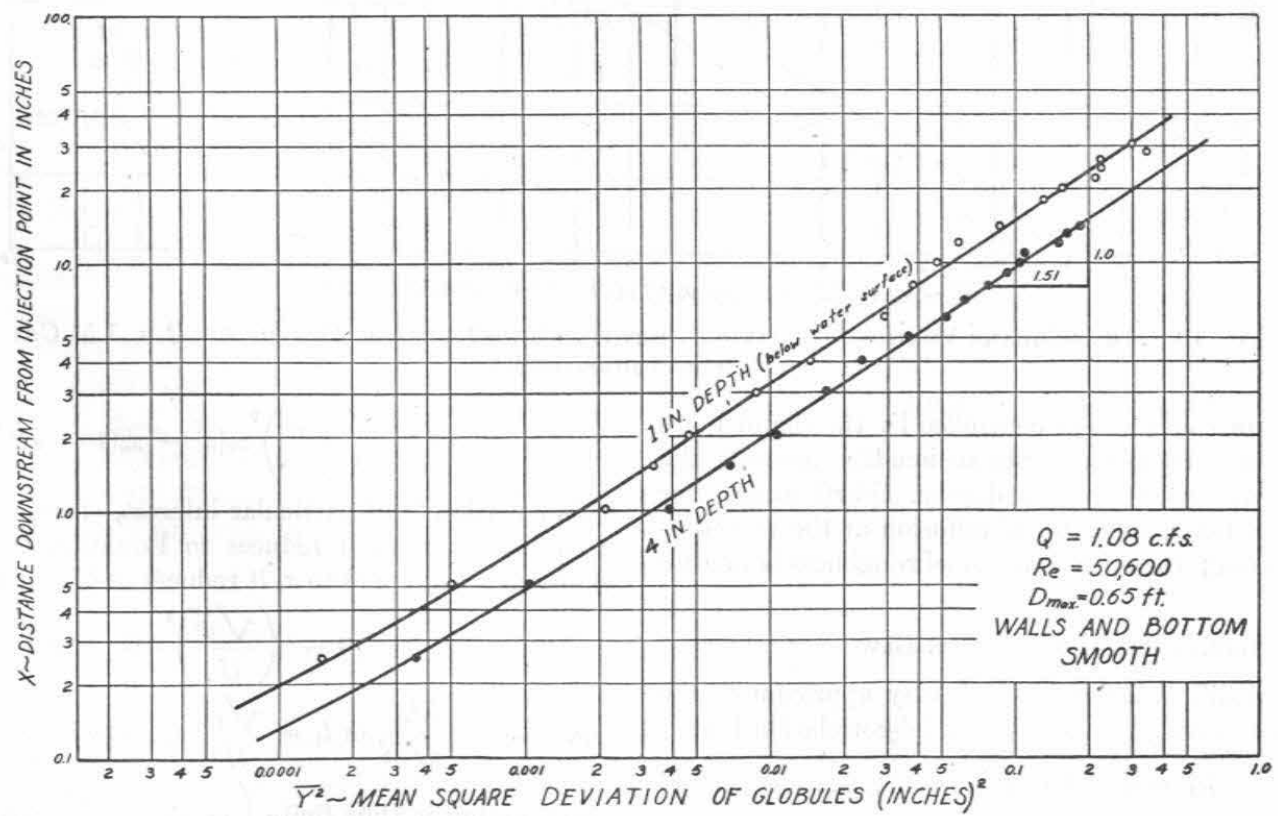

Fig. 8 Mran-Square Vertical Deviation Curves at Two Injection Depths for $Q=1.08$ Cfs (Walls and bottom smooth.) 


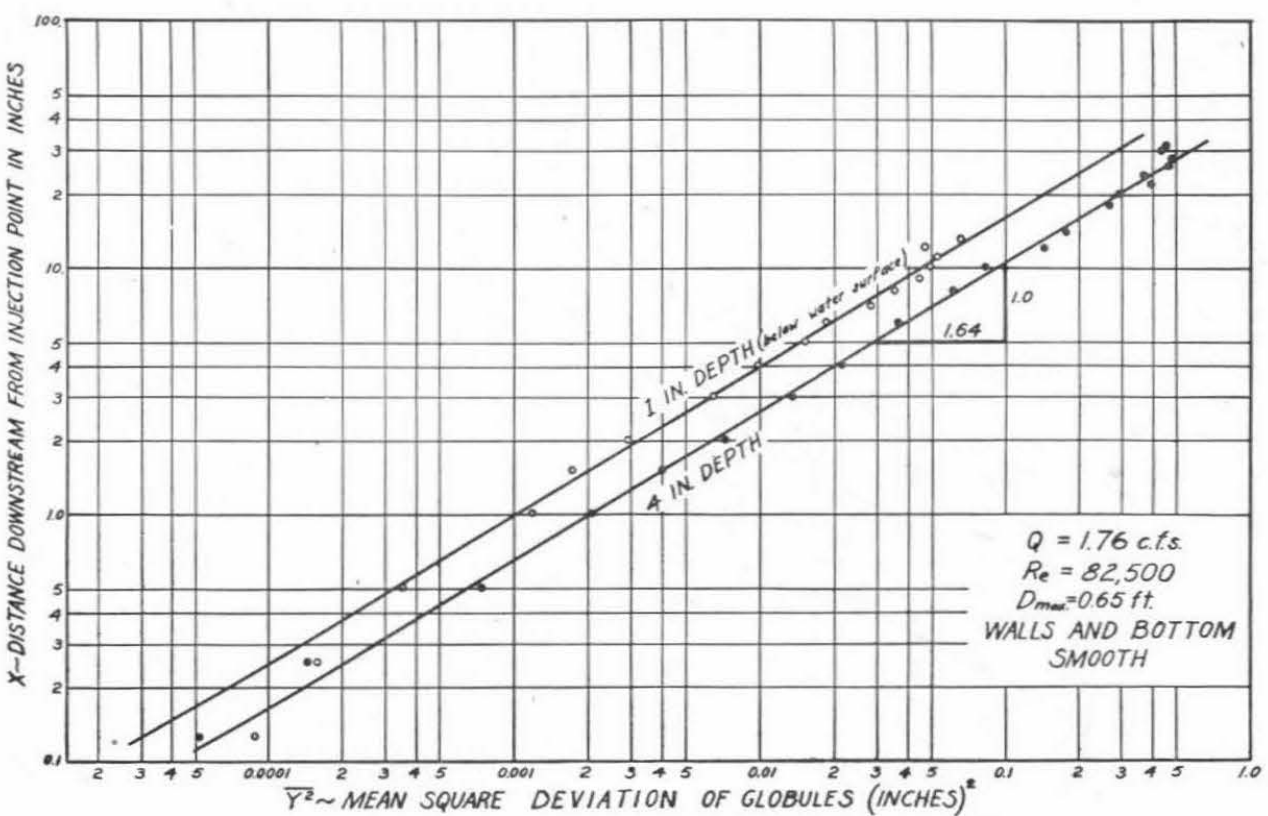

Fig. 9 Mean-Square Vertical Deviation Curves at Two Injection Depths for $Q=1.76$ Cfa (Walls and bottom smooth.)

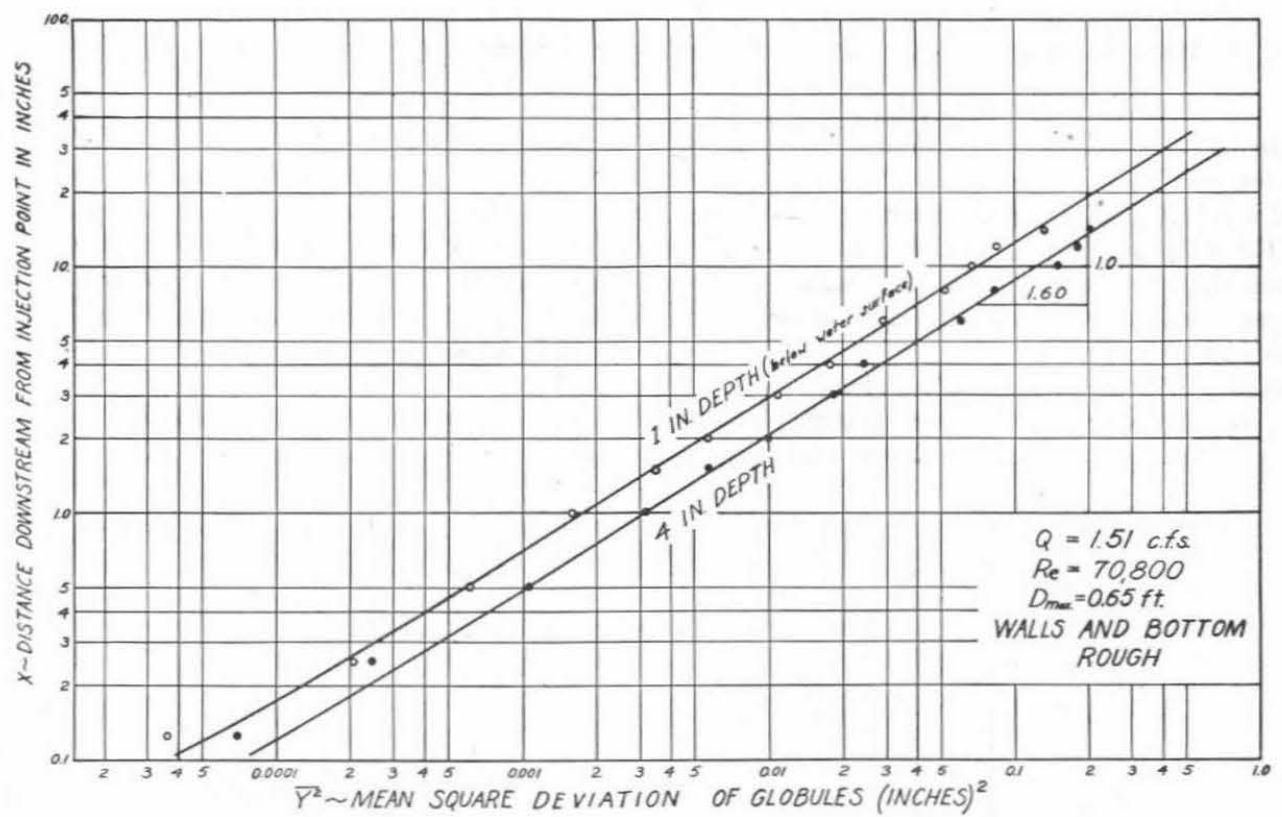

Fig. 10 Mean-Square Vertical Deviation Curves at Two Injection Depths for $Q=1.51$ Cfs (Walls and bottom rough.)

powers for each flow condition, as controlled by the channel, are the same and seems to indicate proportionality between the mean-square deviations at any two depths. Furthermore, for all smooth-channel flows, the ratio of diffusion at the 1- and 4in. depths is the same; the effect of channel roughness is to alter this ratio.

\section{Exponential-Correlation Law}

Another type of diffusion curve which may approximate the data is that corresponding to the exponential-correlation law

$$
R_{\xi}=e^{-x / x_{0}}=e^{-\xi / T_{0}}
$$

suggested by Dryden (13). It is then readily shown that substitution of this law into Equation [22] yields, upon integration

$$
\overline{Y^{2}}=2\left(\frac{\sqrt{\overline{v^{2}}}}{U}\right)^{2} x_{0}\left[x-x_{0}\left(1-e^{-x / x_{0}}\right)\right]
$$

This equation is of particular interest, since, at small values of $x$ compared to $x_{0}$, it reduces to Equation [14], and, at large values of $x$ compared to $x_{0}$ it reduces to Equation [19], viz.

$$
\overline{Y^{2}}=2\left(\frac{\sqrt{\overline{v^{2}}}}{U}\right)^{2} x_{0}\left(x-x_{0}\right)
$$

where $x_{0}=\frac{l_{1} U}{\sqrt{\sqrt{v^{2}}}}$, or $l_{1}=\frac{\sqrt{\overline{v^{2}}}}{U} x_{0}$. The integral of the correlation function is thus finite $\left(=T_{0}=\frac{x_{0}}{U}\right)$, leading to a finite diffusion length and diffusion coefficient. 


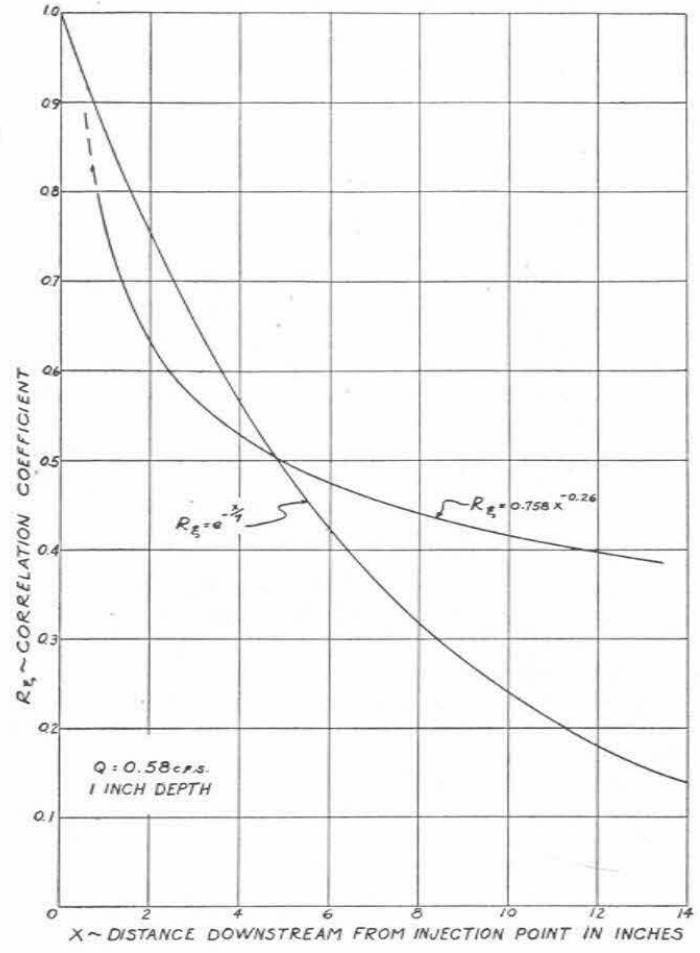

Fig. 11 Typical Power and Exponential-Correlation Curves

Unlike the power law for diffusion, there is no simple form of ${ }^{\circ}$ plotting Equation [25] to give a straight line, so that it is necessary to fit the equation to the data by trial and error, using the usual Cartesian co-ordinate paper; however, from each fit there are obtained both $\sqrt{\overline{v^{2}}} / U$ and $x_{0}$, meaning that it is unnecessary to make a separate determination of $\sqrt{\overline{v^{2}}} / U$ as in the case of the power-correlation law.

Equation [25] has been fitted to the data of only the low flow, $Q=0.58 \mathrm{cfs}$, for the purpose of comparison with power curves. The fitted curves, including the limiting parabolas and asymptotic straight lines, are shown in Fig. 6, along with the power curves. In Fig. 11 are shown two correlation curves at the 1-in. depth (again for the purpose of comparison), one corresponding to Equation [24] and the other to Equation [23], using the values of $x_{0}$ and $\sqrt{\overline{v^{2}}} / U$ obtained from the fitting of Equation [25]. Although it is evident from the plots of the mean-square deviation curves that there is little choice as to which type of curve fits the data, it is nevertheless obvious that the resulting correlation curves are quite different. It may therefore be concluded that the data presented are not of sufficient precision to determine the shape of the correlation curve. If, however, the assumption is made that the curve, corresponding to the exponential-correlation law, represents the data, values of $l_{1}$ and $\sqrt{v^{2}} l_{1}$ can be computed and will appear as shown in Fig. 12.

A dimensionless plot of the data is suggested by Equation [25], which can be written in the following form

$$
\frac{\overline{Y^{2}}}{\left(\frac{\sqrt{\overline{v^{2}}}}{U}\right)^{2} x_{0}{ }^{2}}=\frac{\overline{Y^{2}}}{l_{1}^{2}}=2\left[\frac{x}{x_{0}}-\left(1-e^{-x / x_{0}}\right)\right]
$$

Thus all the data should fall around the single curve, Fig. 13.

It is to be noted that in the fitting process the value of $x_{0}=7$ in. was found to satisfy the data at each injection point in the low flow. This corresponds to the constancy of the power $n$ in the

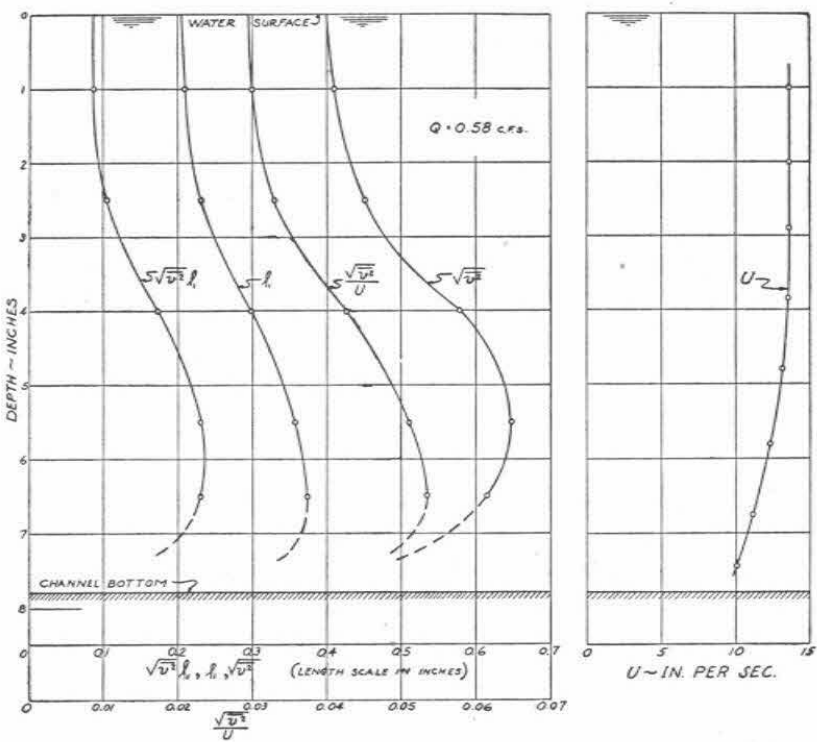

Fig. 12 Diffusion Properties of Turbulence Based on an Exponential-Correlation Law; $Q=0.58$ CFB

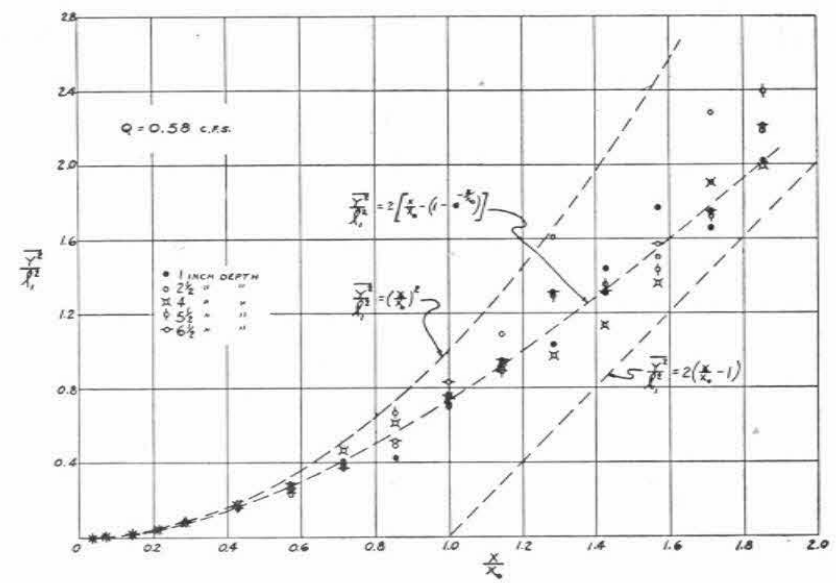

Fig. 13 Dimensionless Plot of Diffusion Using the Data for $Q=0.58 \mathrm{CFS}$

power equation and again indicates proportionality between diffusion at any two depths.

\section{Summary and Conclusion}

An experiment was conducted for the purpose of measuring certain diffusion properties of water flowing in an open channel. By measuring the displacements of immiscible globules, meansquare deviation data were obtained at various depths for three rates of flow in a smooth channel, and for one rate in an artificially roughened channel, all flows having the same total depth. Theory was reviewed to provide the reader with the necessary background for analysis of the data.

It was seen that it was possible to fit either one of two types of curve to the data presented in this paper, one corresponding to a power-correlation law and the other to an exponentialcorrelation law. Since the two curves differed widely in their characteristics, it was concluded that the experimental data, in spite of the fact that about 400 observations were taken for each mean-square de iation point, were not of sufficient precision to warrant the computation of second derivatives and, consequently, the determination of the shape of the correlation curve.

Of the two curves suggested, the one corresponding to the 
exponential-correlation law is of more interest because of its close conformity to the expected nature of the correlation curve, i.e., at a time interval of zero the value of the correlation coefficient is unity, and at a time interval large compared to $T_{0}$ the value of the definite integral of the correlation function approaches a finite quantity. If the assumption is made that such a type of curve represents the data, the parameters of diffusion, $\sqrt{\overline{v^{2}}}$, $l_{1}$, and $\sqrt{\overline{v^{2}}} l_{1}$, along the vertical center line of the flow will appear as shown in Fig. 12.

Despite the appreciable scatter of the mean-square deviation data, it is interesting to note the proportionality of diffusion at all depths.

\section{ACKNOWLEDGMENTS}

This study was conducted under the direction of Robert T. Knapp, Associate Professor of Hydraulic Engineering, California Institute of Technology. The investigation was a project of the Co-Operative Laboratory of the Soil Conservation Service, California Institute of Technology, and the author is greatly indebted to its staff. The work was carried out with the aid of the Work Projects Administration, Official Project No. 65-2-07-58, Work Project No. PS-11496.

\section{BIBLIOGRAPHY}

1 "Application of Statistical Theory of Turbulence to Hydraulic Problems," by A. A. Kalinske and E. R. Van Driest, Proceedings of the Fifth International Congress of Applied Mechanics, Cambridge, Mass., 1938, p. 416.

2 "A Turbulence Indicator Utilizing the Diffusion of Heat," by G. B. Schubauer, National Advisory Committee for Aeronautics, Technical Report no. 524, 1935.

3 "Diffusion Over Distances Ranging From $3 \mathrm{Km}$ to $86 \mathrm{Km}$," by L. F. Richardson and D. Proctor, Memoirs of the Royal Meteorological Society, vol. 1, 1926, p. 1.

4 "Atoms," by J. Perrin, translated by D. L. Hammick, Constable and Co., Ltd., London, Eng., 1920.

5 "An Introduction to the Kinetic Theory of Gases," by Sir James Jeans, Cambridge University Press, 1940, p. 199.

6 "Über die von der molekularkinetischen Theorie der Wärme geforderte Bewegung von in ruhenden Flussigkeiten suspendierten Teilschen," by A. Einstein, Annalen der Physik, vol. 17, 1905, p. 549.

7 "Modern Developments in Fluid Dynamics," composed by the Fluid Motion Panel of the Aeronautical Research Committee and edited by S. Goldstein, Oxford University Press, 1938, p. 205.

8 "Some Experiments on the Transportation of Suspended Load," by Vito A. Vanoni, Transactions of the American Geophysical Union, part 3, 1941, p. 608.

9 "Diffusion by Continuous Movements," by G. I. Taylor,

Proceedings of London Mathematical Society, vol. 20, 1921, p. 196.

10 "Statistical Theory of Turbulence," by G. I. Taylor, Proceedings of the Royal Society of London, series A, vol. 151, 1935, p. 421.

11 "On the Statistical Theory of Isotropic Turbulence," by Th. von Kármán and L. Howarth, Proceedings of the Royal Society of London, series A, vol. 164, 1938, p. 192.

12 "A Theory of Eddy Diffusion in the Atmosphere," by O. G Sutton, Proceedings of Royal Society of London, vol. 135,1932, p. 143.

13 "Turbulence and Diffusion," by Hugh L. Dryden, Industrial and Engineering Chemistry, vol. 31, 1939, p. 416. 